\title{
Atividade de Mansonia spp. (Mansoniini, Culicidae) em fragmento florestal na área urbana de Curitiba, Paraná, Brasil ${ }^{1}$
}

\author{
Mario Antonio Navarro-Silva ${ }^{2}$, Andreia Aparecida Barbosa ${ }^{2,3}$ \& Daniéla Calado ${ }^{2,3}$ \\ ${ }^{1}$ Contribuição número 1461 do Departamento de Zoologia, Universidade Federal do Paraná. \\ ${ }^{2}$ Departamento de Zoologia, Universidade Federal do Paraná. Caixa Postal 19020, 81531-980 Curitiba, Paraná, Brasil. \\ E-mail: mnavarro@ufpr.br \\ 3 Bolsista de Doutorado do CNPq.E-mail: deiaguel@ufpr.br, danielacalado@hotmail.com
}

\begin{abstract}
Mansonia spp. (Mansoniini, Culicidae) activity in a restrict forest inside Curitiba urban area (Paraná, Brazil). Vegetation islands surrounded by urban areas can provide favourable conditions to the development of Culicidae population. In Regional Iguaçu Park (Curitiba, Paraná) there are many lakes with aquatic plants that can be used by Mansoniini immatures. The aim of this investigation was to know the Mansonia Blanchard, 1901 species that are blood feeding during the day in a wood area inside the park. The mosquitoes captures were carried out from september, 1999 to august, 2000. Three species that belong to Mansonia were identified, and the percentages were: $74.7 \%$ de M. fonsecai (Pinto, 1932), 10.7\% de M. wilsoni (Barreto \& Coutinho, 1944) e $8.3 \%$ de M. tittilans (Walker, 1848). By the analysis of the activity hour, the species were collected during all the day in the same rhythm, and the hours mean were 9.5, 1.3 e 1.0, respectively.

KEY WORDS. Mansonia wilsoni, Mansonia tittilans, Mansonia fonsecai, vectors.
\end{abstract}

RESUMO. Fragmentos de mata no cenário urbano podem reunir condições favoráveis à reprodução de espécies de Culicidae. No Parque Regional do Iguaçu (Curitiba, Paraná) existe uma série de cavas ou lagoas contendo vegetação aquática que pode ser utilizada pelos imaturos das espécies de Mansoniini.A investigação foi desenvolvida com objetivo de conhecer as espécies de Mansonia Blanchard, 1901 que exercem hematofagia no período diurno em área de mata no interior do parque. As coletas dos mosquitos foram efetuadas de setembro de 1999 a agosto de 2000. Três espécies de Mansonia foram identificadas, com os seguintes percentuais relativos: $74,7 \%$ de $M$. fonsecai (Pinto, 1932), 10,7\% de M. wilsoni (Barreto \& Coutinho, 1944) e 8,3\% de M. tittilans (Walker, 1848). Quanto ao horário de atividade, as espécies ocorreram durante todo o dia em ritmo similar, com as seguintes médias horárias 9,5, 1,3 e 1,0 para M. fonsecai, M. wilsoni e M. tittilans, respectivamente.

PALAVRAS CHAVE. Mansonia wilsoni, Mansonia tittilans, Mansonia fonsecai, vetores.

Fragmentos de mata em área urbana, desempenham diversas funções ecológicas. No caso específico do município de Curitiba, algumas áreas foram projetadas para evitar a ocorrência de enchentes em áreas consideradas de risco ou destinadas ao lazer da população. A área do Parque Regional do Iguaçu é formada por um mosaico de espaços onde estão inseridos, por exemplo, o Zoológico Municipal e uma área de fragmento florestal com instalações destinadas às atividades de Educação Ambiental.

Apesar de sua importância estratégica na construção de ambientes comunitários de lazer e proteção ambiental, fragmentos do ambiente natural quando inseridos em áreas alteradas, podem reunir condições favoráveis aos processos adaptativos de insetos em suas relações com a população humana, como observado por Taipe-Lagos \& Natal (2003).
Por outro lado, as áreas contíguas daquelas destinadas à preservação também devem receber atenção e manutenção. Na área do Parque Regional do Iguaçu encontra-se uma série de cavas ou lagos que abrigam extensa cobertura de vegetação aquática flutuante e que constituem habitats propícios ao desenvolvimento de imaturos de Mansoniini. As fêmeas deste táxon são hematófagas exófilas, agressivas, vorazes e têm elevada capacidade de dispersão. Determinadas espécies já foram encontradas naturalmente infectadas por diversos arbovírus (ForatTini 2002, Dorvillé 1995).

O presente estudo centralizou a atenção na atividade das espécies de Masoniini em fragmento florestal com objetivo de verificar a freqüência e abundância de exemplares sobre hospedeiro humano.

Revista Brasileira de Zoologia 21 (2): 243-247, junho 2004 


\section{MATERIAL E MÉTODOS}

O Parque Regional do Iguaçu localiza-se na região sudeste do Município de Curitiba (Estado do Paraná), entre as coordenadas $25^{\circ} 25^{\prime} 55^{\prime \prime} \mathrm{S}$ e $49^{\circ} 12^{\prime} 05^{\prime \prime} \mathrm{W}$. Criado na década de 70 para a contenção de cheias nas regiões sul e sudeste de Curitiba, está implantado na Bacia do Rio Iguaçu e conta com uma área total de $8.264 .316 \mathrm{~m}^{2}$. É considerado o maior parque regional do país situado em centro urbano.

Sua composição florística é representada por remanescente de Floresta Ombrófila Mista e apresenta quatro ecossistemas distintos: as várzeas ou campos de baixadas, desenvolvendo-se sobre solos sujeitos a inundações periódicas e permanentes; os campos limpos, representados por gramíneas; os capões, que ocorrem ao redor das nascentes e em depressões úmidas e com composição florística característica das florestas com Araucaria Juss. (Araucariaceae) e as matas ciliares cuja espécie dominante Sebastiana commersoniana (Baill.) Smith \& Downs (Euphorbiaceae) perfaz $80 \%$ de sua composição.

A distribuição destas formações vegetais, ao longo do parque, é desigual e dispersa em conseqüência da expansão urbana e da ação antrópica. O ambiente encontra-se modificado pelas inúmeras cavas e lagoas formadas pela atividade de extração de argila e areia ao longo do leito do Rio Iguaçu. Atualmente, o Parque Regional do Iguaçu abriga o Zoológico Municipal de Curitiba e constitui uma Área de Proteção Ambiental.

As capturas de culicídeos adultos foram realizadas em área de mata destinada ao acantonamento. Este habitat é alvo de visitas de estudantes da rede pública de ensino que participam do "Projeto de Acantonamento Ecológico". As coletas de culicídeos adultos foram efetuadas mensalmente, de setembro de 1999 a agosto de 2000, em dois períodos: a) pela manhã das 9:00 as 12:00 h e b) à tarde das 13:00 as 16:00 h. Cada período foi subdividido em intervalos de 30 minutos, totalizando 72 horas de coleta.

As capturas foram realizadas ao nível do solo, distante 150 metros da borda da mata, utilizando a técnica pouso-homem (human landing) conforme ForatTini et al. (2000), com um coletor. Os exemplares capturados encontram-se depositados na Coleção Entomológica Pe. Jesus Santiago Moure (DZUP), Departamento de Zoologia, Universidade Federal do Paraná.

Para comparação entre a flutuação populacional das espécies coletadas e as variáveis climáticas, foram obtidas planilhas de observações meteorológicas da estação número 83.842 do Instituto Meteorológico do Paraná (SIMEPAR), localizada no Centro Politécnico da Universidade Federal do Paraná.

\section{RESULTADOS E DISCUSSÃO}

No interior do fragmento de mata foram coletados 916 exemplares de Mansonia Blanchard, 1901, dos quais 8,3\% pertencentes a M. tittilans (Walker, 1848), 10,7\% M. wilsoni (Barreto \& Coutinho, 1944) e $74,7 \%$ M. fonsecai (Pinto, 1932). O restante dos exemplares (6,3\%) de Mansonia não foi possível identificar até o presente momento (Fig. 1 e Tab. I).

Revista Brasileira de Zoologia 21 (2): 243-247, junho 2004

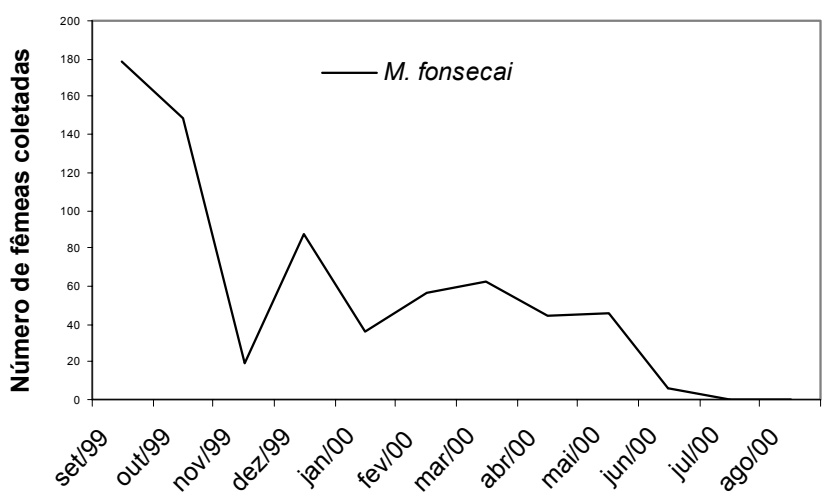

A
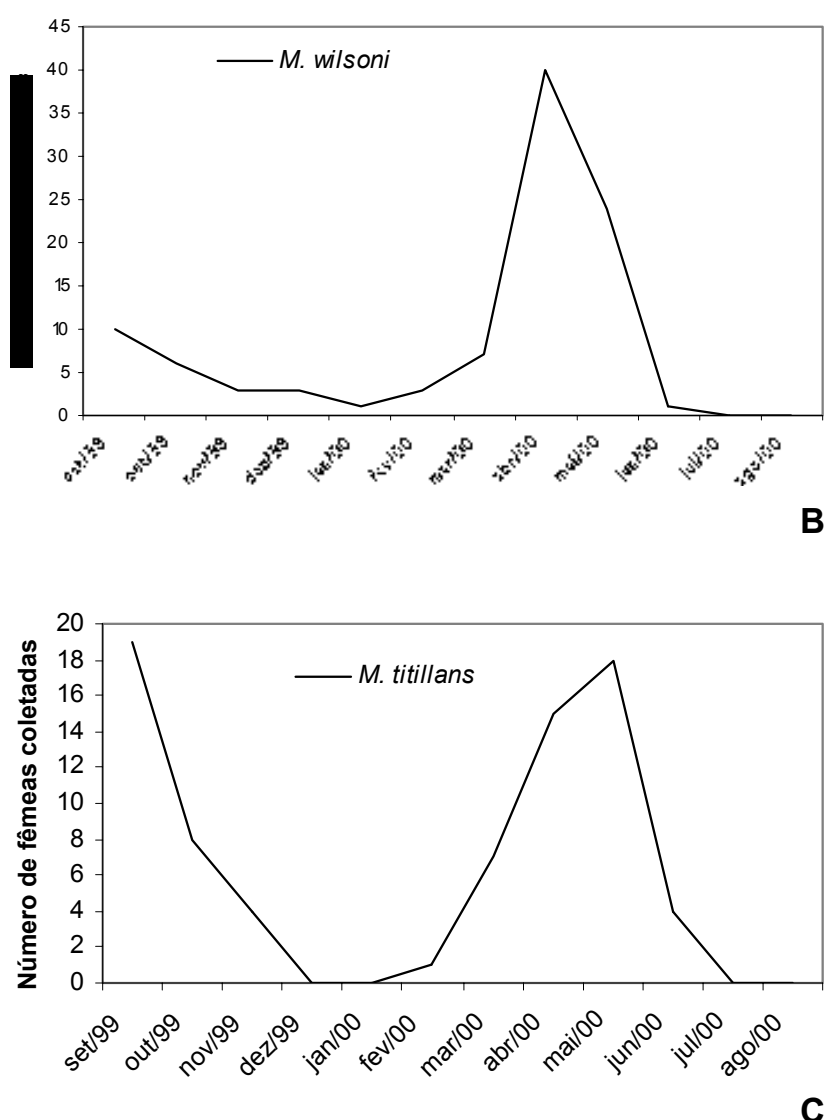

Figura 1. Distribuição mensal de Mansonia fonsecai (A), M. wilsoni (B) e M. tittilans (C) capturadas através da técnica pouso-homem, em área do Parque Regional do Iguaçu, Curitiba, Paraná, setembro de 1999 a agosto de 2000.

O período de setembro de 1999 a agosto de 2002 foi marcado por inverno com temperatura mínima média de $6^{\circ} \mathrm{C}$ e verão com temperatura média superior a $25^{\circ} \mathrm{C}$. A umidade 
Tabela I. Número de fêmeas de Mansonia capturadas em cada intervalo horário, através da técnica pouso-homem, em área do Parque Regional do Iguaçu, Curitiba, Paraná, no período de setembro de 1999 a agosto de 2000.

\begin{tabular}{|c|c|c|c|c|c|}
\hline Intervalos de horários & Mansonia fonsecai & Mansonia wilsoni & Mansonia tittilans & Mansonia spp. & Total \\
\hline \multicolumn{6}{|l|}{ Período matutino } \\
\hline 09:00/09:30 & 165 & 22 & 18 & 32 & 237 \\
\hline 09:30/10:00 & 60 & 10 & 7 & 1 & 78 \\
\hline 10:00/10:30 & 67 & 7 & 6 & 3 & 83 \\
\hline 10:30/11:00 & 35 & 6 & 6 & 3 & 50 \\
\hline $11: 00 / 11: 30$ & 20 & 6 & 5 & 0 & 31 \\
\hline 11:30/12:00 & 39 & 5 & 6 & 0 & 50 \\
\hline \multicolumn{6}{|l|}{ Período vespertino } \\
\hline 13:00/13:30 & 71 & 20 & 6 & 7 & 104 \\
\hline $13: 30 / 14: 00$ & 46 & 5 & 4 & 7 & 62 \\
\hline $14: 00 / 14: 30$ & 33 & 6 & 4 & 0 & 43 \\
\hline $14: 30 / 15: 00$ & 64 & 4 & 7 & 1 & 76 \\
\hline $15: 00 / 15: 30$ & 52 & 2 & 2 & 1 & 57 \\
\hline $15: 30 / 16: 00$ & 32 & 5 & 5 & 3 & 45 \\
\hline Média Horária & 9,5 & 1,3 & 1,0 & 0,8 & 12,7 \\
\hline Total de fêmeas & 684 & 98 & 76 & 58 & 916 \\
\hline
\end{tabular}

relativa do ar oscilou entre 70 e $80 \%$. Em relação à precipitação pluviométrica, houve queda acentuada em abril e maio em comparação com os demais meses do estudo.

Não foi observada correlação significativa entre a ocorrência das espécies de Mansonia e a variável temperatura (Tab. II). Entretanto, nos meses de julho e agosto, em que foram observadas as temperaturas mínimas médias mais baixas $\left(6-8^{\circ} \mathrm{C}\right)$, nenhuma fêmea foi capturada. Possivelmente, porque sob temperaturas muito baixas, como as registradas em Curitiba durante $o$ inverno, a atividade e sobrevivência de adultos tenham sido reduzidas (Fig. 2).

Quanto à precipitação pluviométrica, a correlação foi negativa entre a quantidade média de chuvas e a atividade hematofágica de M. wilsoni e M. tittilans (Tab. III). Da mesma forma que a temperatura pode influenciar a atividade dos culicídeos adultos, a precipitação pluviométrica pode levar à redução da atividade de procura por hospedeiros e por criadouros, pois dificulta a atividade de vôo e/ou diminui a sobrevivência dos indivíduos. Além disso, os canais artificiais de drenagem poderiam ser responsáveis pelo transporte da vegetação aquática e dispersão de imaturos para outras áreas, reduzindo assim a população de adultos.

Embora os representantes de Mansonia apresentem atividade crepuscular e noturna (ForatTINI, 2002), no presente estudo foi observada a presença de espécies desse gênero durante o período diurno (Tab. I). Aproximadamente 37\% dos exemplares foram coletados nos primeiros intervalos de amostragens
Tabela II. Coeficiente de Correlação Linear entre as médias mensais de temperatura máxima, média e mínima e o número de fêmeas de Mansonia capturadas através da técnica pouso-homem, em área do Parque Regional do Iguaçu, Curitiba, Paraná, setembro de 1999 a agosto de 2000 .

\begin{tabular}{cccc}
\hline Espécies & $\begin{array}{c}\text { Temperatura } \\
\text { máxima }\left({ }^{\circ} \mathrm{C}\right)\end{array}$ & $\begin{array}{c}\text { Temperatura } \\
\text { média }\left({ }^{\circ} \mathrm{C}\right)\end{array}$ & $\begin{array}{c}\text { Temperatura } \\
\text { mínima }\left({ }^{\circ} \mathrm{C}\right)\end{array}$ \\
\hline Mansonia fonsecai & $-0,082$ & 0,156 & 0,216 \\
Mansonia wilsoni & 0,135 & 0,083 & 0,045 \\
Mansonia tittilans & $-0,135$ & $-0,130$ & $-0,133$ \\
Mansonia spp. & 0,459 & 0,504 & 0,544 \\
\hline
\end{tabular}

Tabela III. Coeficiente de Correlação Linear entre as médias mensais de precipitação pluviométrica, umidade relativa do ar máxima, média e mínima e o número de fêmeas de Mansonia capturadas através da técnica pouso-homem, em área do Parque Regional do Iguaçu, Curitiba, Paraná, setembro de 1999 a agosto de 2000. (UR) Umidade relativa do ar, (PP) precipitação pluviométrica.

\begin{tabular}{lcccc}
\hline \multirow{2}{*}{ Espécies } & \multicolumn{3}{c}{ UR (\%) } & $\begin{array}{c}\text { PP média } \\
(\mathrm{mm})\end{array}$ \\
\cline { 2 - 4 } & Máxima & Média & Mínima & 0,243 \\
\hline Mansonia fonsecai & 0,153 & 0,352 & 0,382 & $0,0,727$ \\
Mansonia wilsoni & 0,050 & $-0,243$ & $-0,403$ & $-0,727$ \\
Mansonia tittilans & 0,027 & $-0,192$ & $-0,239$ & $-0,522$ \\
Mansonia spp & 0,188 & 0,493 & 0,425 & 0,288 \\
\hline
\end{tabular}

Revista Brasileira de Zoologia 21 (2): 243-247, junho 2004 

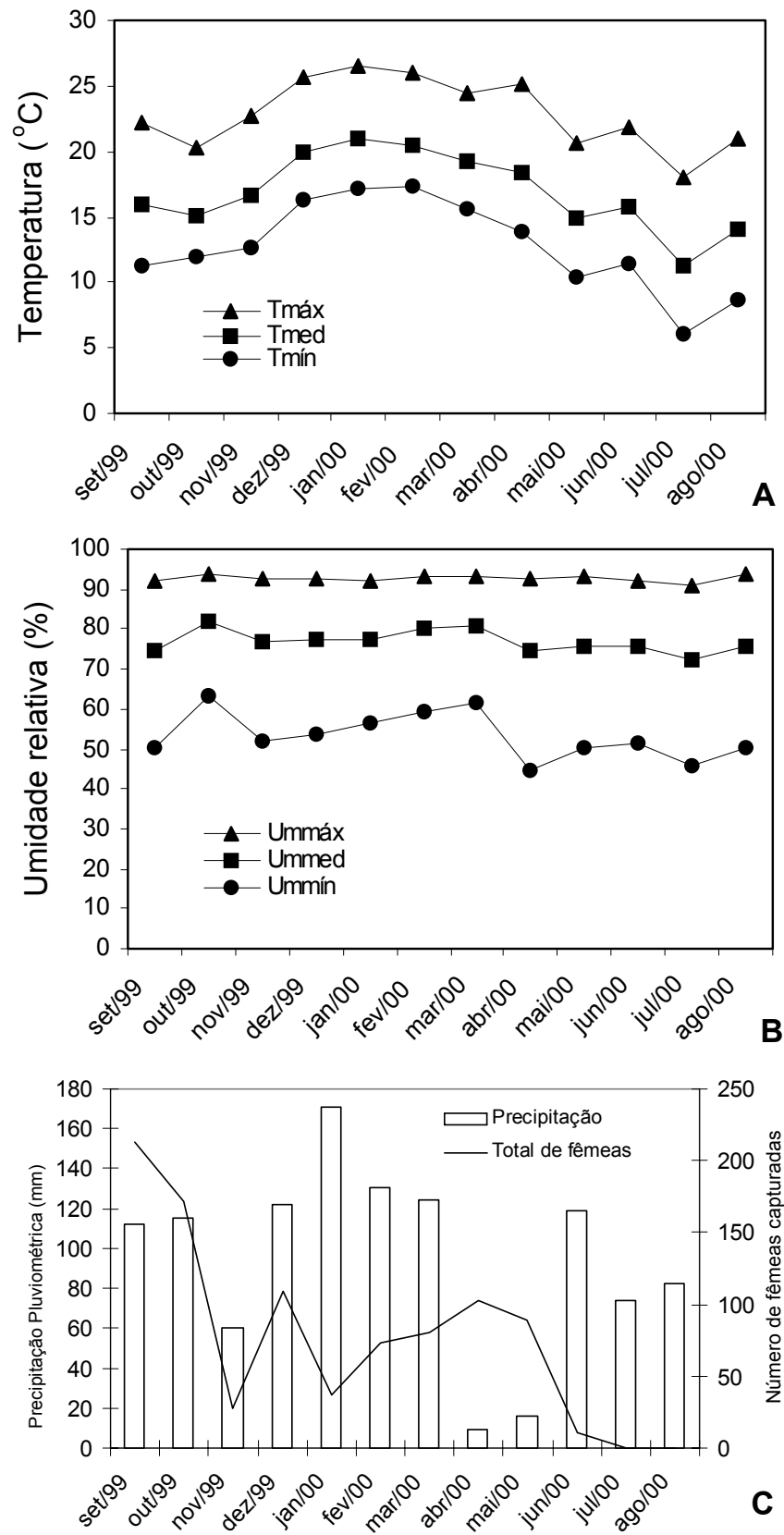

Figura 2. Distribuição mensal da temperatura (A), umidade relativa do $\operatorname{ar}(B)$, precipitação pluviométrica e número de espécies de Mansonia coletadas (C) em área do Parque Regional do Iguaçu, Curitiba, Paraná, setembro de 1999 a agosto de 2000.

(9:00-9:30 h e 13:00-13:30 h), confirmando a agressividade com que as espécies de Mansonia buscam seus hospedeiros (Forattini 2002, Lopes et al. 1995). Por tratar-se de área intensamente visitada e circundada por habitações, a característica agressiva ali- ada ao papel como possíveis vetores de arbovírus assume importante significado epidemiológico, pois indicam maior chance de contato homem-vetor e a possível veiculação de agentes patogênicos. Destaca-se ainda o incômodo causado por estes culicídeos através da picada.

Os resultados obtidos no presente estudo, refletem a importância da ação antrópica sobre o ambiente, que pode ser traduzida pela presença do gênero Mansonia próximo ao ambiente urbano. Embora as espécies desse gênero possam ser consideradas predominantemente exófilas e florestais (ForATTINI 2002), a atividade antrópica pode gerar condições adequadas à presença desses culicídeos próximo aos centros urbanos.

A formação de cavas e lagoas através da atividade extrativista, no Parque Regional em estudo, favoreceu o surgimento de inúmeros micro-habitats, especialmente aqueles representados pela vegetação aquática e que podem abrigar imaturos das espécies de Mansoniini. Podem ser observados densos conjuntos de vegetação aquática, especialmente de Pistia stratiotes L. (Araceae, alface-d'água) e também representantes de Eichornia crassipes (Mart.) Solms (Pontederiaceae, aguapé), Heteranthera reniformis Ruiz \& Pavón (Pontederiaceae, hortelã-do-brejo), Ponteria cordata L. (Pontederiaceae, rainha-dos-lagos), Typha domingensis Pers. (Typhaceae, taboa) e Echynocloa polystachya (Kunth) Hitchc. (Poaceae, capim-capivara). A ocorrência de grande diversidade de vegetais aquáticos poderá favorecer a ocorrência de outras espécies de Culicidae, bem como a manutenção e/ou aumento das populações já existentes. Em altas densidades, os representantes de Mansonia poderão causar incômodos aos visitantes do parque e aos habitantes das áreas circunvizinhas, como já verificado por Lopes et al. (1995).

Embora o Parque represente área restrita no interior da estrutura urbana, apresenta considerável cobertura vegetal em condições de sustentar diversidade de aves residentes permenentes (não migratórias) e eventualmente as sazonais (Anjos \& Laroca, 1989, Pereira et al., 2001), ambas apresentando-se como fonte potencial para o exercício da hematofagia das fêmeas de Mansonia. Na continuidade do parque encontramos o Zoológico Municipal que abriga fauna em cativeiro bastante diversificada. A presença destes animais na área e o processo de migração de muitas espécies de aves ao longo do ano, podem potencializar a introdução de agentes patogênicos na área, tendo em vista o ecletismo de algumas espécies do gênero Mansonia quanto aos hospedeiros e o alto grau de zoofilia (LouRENÇO-DE-Oliveira et al. 1986).

A manutenção adequada dos espaços urbanos é desafio constante e deve ser perseguido com objetivo de melhorar a qualidade de vida das populações urbanas. Dentro do item manutenção, também devem ser implementadas ações que visem monitorar as populações de Culicidae, além daquelas da rotina epidemiológica como Aedes aegypti Linnaeus, 1762 e Aedes albopictus Skuse, 1894, visto que o melhor conhecimento das relações entre as espécies vetoras e as potencialmente vetoras em ambiente marcado pela atuação antrópica podem 
ampliar o conhecimento e as estratégias a serem utilizadas no controle e prevenção de possíveis introduções de agentes patogênicos nas áreas urbanizadas.

\section{AGRADECIMENTOS}

Ao Instituto Meteorológico do Paraná (SIMEPAR) pelas planilhas meteorológicas.

À diretora do Acantonamento do Parque Regional do Iguaçu, Marcia Frasson, pela possibilidade de realização deste estudo na área do parque.

\section{REFERÊNCIAS BIBLIOGRÁFICAS}

Anjos, L. Dos \& S. Laroca. 1989. Abundância relativa e diversidade específica em duas comunidades urbanas de aves de Curitiba (Sul do Brasil). Arquivos de Biologia e Tecnologia, Curitiba, 32 (4): 637-643.

DoRvillé, L.F.M. 1995. Composição e aspectos da biologia da fauna de mosquitos (Diptera, Culicidae) da Restinga de Barra de Maricá (RJ). Revista Brasileira de Entomologia, Curitiba, 39 (1): 203-219.

Forattini, O.P.; I. Kakitani; R.L.C. dos Santos; K. Kobayashi; H.M.
Ueno \& Z. Fernandez. 2000. Comportamento de Aedes albopictus e Ae. scapularis adultos (Diptera: Culicidae) no Sudeste do Brasil. Revista de Saúde Pública, São Paulo, 34 (5): 461-467.

Forattini, O.P. 2002. Culicidologia Médica. São Paulo, EDUSP, $864 p$.

Lopes, J.; V.D.R.B. de Oliveira \& F.J. De A. Oliveira. 1995. Predominância de Mansonia tittilans (Walker, 1848) (Diptera: Culicidae) na área urbana do município de Londrina-PR, sul do Brasil. Semina, Londrina, 16 (2): 254-259.

Lourenço-De-Oliveira, R.; R. Heyden \& T.F. dA Silva. 1986. Alguns aspectos da ecologia dos mosquitos (Diptera, Culicidae) de uma área de planície (Granjas Calábria), em Jacarepaguá, Rio de Janeiro. V. Criadouros. Memórias do Instituto Oswaldo Cruz, Rio de Janeiro, 81 (3): 265-271.

Pereira, L.E.; A. Suzuki; T.L.M. Coimbra, R.P.de Souza \& E.L.B. CHAMELEt. 2001. Arbovírus Ilhéus em aves silvestres (Sporophila caerulescens e Molothurus bonariensis). Revista de Saúde Pública, São Paulo, 35 (2): 119-123

Taipes-Lagos, C.B. \& D. Natal. 2003. Abundância de culicídeos em área metropolitana preservada e suas implicações epidemiológicas. Revista de Saúde Pública, São Paulo, 37 (3): 275-279.

Recebido em 14.XI.2003; aceito em 11.V.2004. 